\title{
High-sensitive and temperature-self-calibrated tilted fiber grating biological sensing probe
}

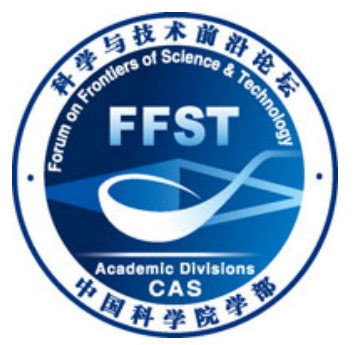

\author{
LIU Fu ${ }^{1}$, GUO Tuan ${ }^{1 *}$, LIU JianGuo ${ }^{2}$, ZHU XiaoYang ${ }^{3}$, LIU Yu $^{3}$, GUAN BaiOu ${ }^{1} \&$ \\ ALBERT Jacques ${ }^{4}$
}

${ }^{1}$ Institute of Photonics Technology, Jinan University, Guangzhou 510632, China;

${ }^{2}$ Institute of Semiconductors, Chinese Academy of Sciences, Beijing 100083, China;

${ }^{3}$ Department of Biochemistry, Medical School, Jinan University, Guangzhou 510632, China;

${ }^{4}$ Department of Electronics, Carleton University, Ottawa K1S 5B6, Canada

Received August 6, 2012; accepted September 19, 2012; published online April 8, 2013

\begin{abstract}
High sensitivity biological sample measurement has been achieved by using a $10^{\circ}$ tilted fiber Bragg grating sensing probe. Human acute leukemia cells with different intracellular densities were clearly discriminated by identifying their slight refraction index (RI) perturbations in the range from 1.3342 to 1.3344 , combining with a temperature self-calibration property. We studied the relationship between the intracellular density of cells (S50 and S60) and their RIs, the experimental results provide a potential way to verify the hypothesis for "density alteration in non-physiological cells (DANCE)".
\end{abstract}

biosensor, tilted fiber grating, refractometer

Citation: $\quad$ Liu F, Guo T, Liu J G, et al. High-sensitive and temperature-self-calibrated tilted fiber grating biological sensing probe. Chin Sci Bull, 2013, 58: 2611-2615, doi: 10.1007/s11434-013-5724-3

The tilted fiber Bragg gratings (TFBGs) [1,2], due to the induction of a tilted angle between the UV laser beam and the fiber axis (otherwise similar to the normal straight fiber grating), provides an effective way which couples the input light from the forward-propagating core mode to backwardpropagating cladding modes [3-6]. Because of these cladding excitations, TFBG shows good sensitivity to surrounding refraction index (SRI) change [7-11]. With the increase of tilt angle, the envelope of the cladding-mode resonance shifts towards shorter wavelength, which shows a much improved RI sensitivity as these cladding modes propagate close to the interface between the cladding mode and the outside media. The dominant cladding modes of TFBG cover the very important region near 1.3 which can be used to measure the water and water-like solutions. Therefore, TFBGs provide a good choice for biochemical sensing. At the same time, all the wavelength resonances of a TFBG have the same temperature dependence (they shift by $\sim 10$

*Corresponding author (email: tuanguo@jnu.edu.cn) $\mathrm{pm} /{ }^{\circ} \mathrm{C}$ ), so we could only consider the relative wavelength shifts and the temperature cross sensitivity over RI measurement can be definitely eliminated [12].

In this paper, the discrimination of a group of biological samples, named S50 and S60 (corresponding to cells with different intracellular densities), which were separated from human acute leukemia cell line (K562) [13-18] by using discontinuous sucrose gradient centrifugation (DSGC) [19,20], has been achieved through a high sensitivity RI measurement by using a $10^{\circ}$ TFBG sensing probe. By comparing the slight RI difference between S50 and S60 (ranging from 1.3343 to1.3344), we studied the relationship between the intracellular density of cells and their RI, which provide a potential way to verify the hypothesis for "density alteration in non-physiological cells (DANCE)" [21,22].

\section{Theory of mode coupling in tilted fiber grating}

TFBGs are fabricated by using phase mask method from a 
permanent refractive index change by induced a tilted angle between the UV laser beam and the fiber axis. In order to obtain the desired tilt angle, we rotated the phase mask around the axis of the writing beam. As shown in Figure 1, TFBGs provide an effective way to couple the input light from the forward-propagating core mode to backward-propagating cladding modes which can be detected in transmission spectra.

Coupling input light to backward-propagating core mode only, the incident and reflected light of FBG are limited to propagate in the fiber core. The transmission resonances meeting Bragg condition can be expressed as

$$
\lambda_{\text {Bragg }}=2 n_{\text {eff,core }} \Lambda
$$

where $n_{\text {eff,core }}$ is the effective index of the core mode, and $\Lambda$ is the period of the interference pattern. As for TFBG, the $\Lambda$ can be considered as

$$
\Lambda_{g}=\Lambda / \cos \theta
$$

where $\theta$ is the tilt angle of the grating planes. By the same way, the Bragg reflection and cladding modes resonance wavelengths $\left(\lambda_{\text {Bragg }}\right.$ and $\lambda_{i}$ ) of TFBG are decided by a phase-matching condition and can be considered as

$$
\begin{gathered}
\lambda_{\text {Bragg }}=2 n_{\text {eff,core }} \Lambda_{g}, \\
\lambda_{i}=\left(n_{\text {eff,core }}+n_{\text {eff,clad }, i}\right) \Lambda_{g},
\end{gathered}
$$

where $n_{\text {eff,clad, } i}$ is the effective index of the cladding modes, in which $i$ indicates the order of cladding modes. We considered the Bragg and cladding mode wavelength shifts $\left(\Delta \lambda_{\text {Bragg }}, \Delta \lambda_{i}\right)$ which are caused by SRI $\left(\Delta n_{s}\right)$ changes. From eqs. (3) and (4), the wavelength shifts $\Delta \lambda_{\text {Bragg }}$ and $\Delta \lambda_{i}$ can be expressed as

$$
\Delta \lambda_{\mathrm{Bragg}}=\left(2 \frac{\Lambda}{\cos \theta} \frac{\partial n_{\mathrm{eff}, \mathrm{core}}}{\partial n_{s}}+2 \frac{n_{\mathrm{eff}, \mathrm{core}}}{\cos \theta} \frac{\partial \Lambda}{\partial n_{s}}\right) \Delta n_{s},
$$

$$
\Delta \lambda_{i}=\left(\frac{\Lambda}{\cos \theta} \frac{\partial\left(n_{\text {eff,core }}+n_{\text {eff,clad }, i}\right)}{\partial n_{s}}+\frac{n_{\text {eff,core }}+n_{\text {eff,clad }, i}}{\cos \theta} \frac{\partial \Lambda}{\partial n_{s}}\right) \Delta n_{s} .
$$

Due to $\partial \Lambda / \partial n_{s}=0$, and noting that $\partial n_{\text {eff,core }} / \partial n_{s}=0$ (the standard single mode telecommunication fibers), we obtain

$$
\begin{gathered}
\Delta \lambda_{\text {Bragg }}=0, \\
\Delta \lambda_{i}=\frac{\Lambda}{\cos \theta} \frac{\partial n_{\text {eff,clad }, i}}{\partial n_{s}} \Delta n_{s} .
\end{gathered}
$$

We can conclude that the cladding mode resonances shift depend on the SRI perturbations, meanwhile, the dependence is scaled by the dispersion of the cladding modes. When the SRI matches the effective index of one of the cladding modes, some cladding mode resonances gradually disappear as these mode couple to the outside. The envelope of the cladding modes shifts toward long-wavelength due to the SRI increases. Therefore, the RI measurement can be obtained by this way. Furthermore, the higher order cladding modes are excited by the increasing of the tilt angle. The cladding mode resonances of the $10^{\circ}$ TFBG cover the very important region near 1.33 , so they can be used to measure the water and water-like biological solutions.

\section{Results and discussion}

\subsection{Experimental setup}

The experimental setup for biochemical solutions measurement based on a $10^{\circ}$ TFBG is illustrated in Figure 2. Here, the sensing TFBG was lighted by a broadband source (BBS) and its transmission spectrum was monitored by an optical spectrum analyzer (OSA) with a resolution of $0.02 \mathrm{~nm}$.

\subsection{RI responding characteristics}

As shown in Figure 3, a set of sucrose solutions with different

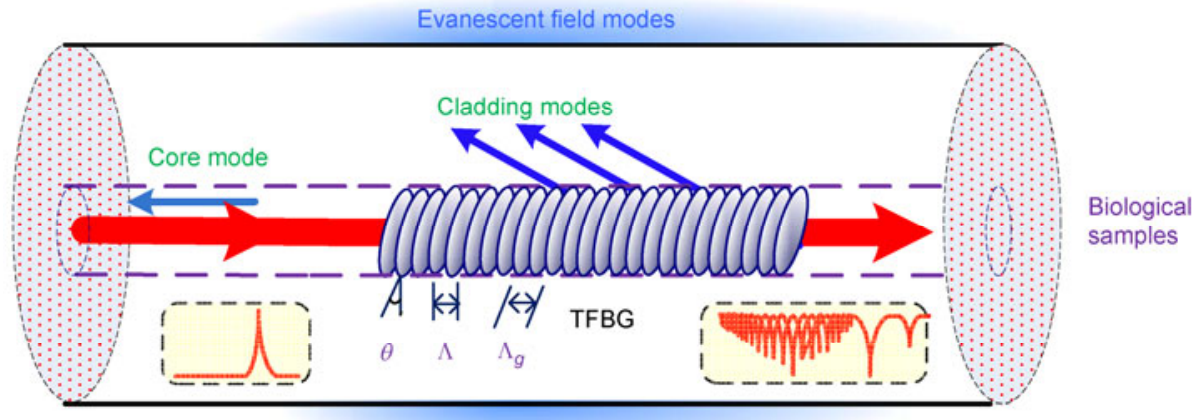

Figure 1 The schematic diagram of TFBG biological sensor. 


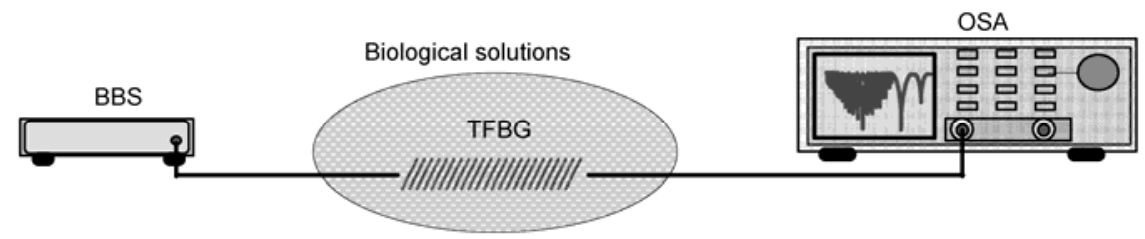

Figure 2 Experimental setup of biological sample measurement.
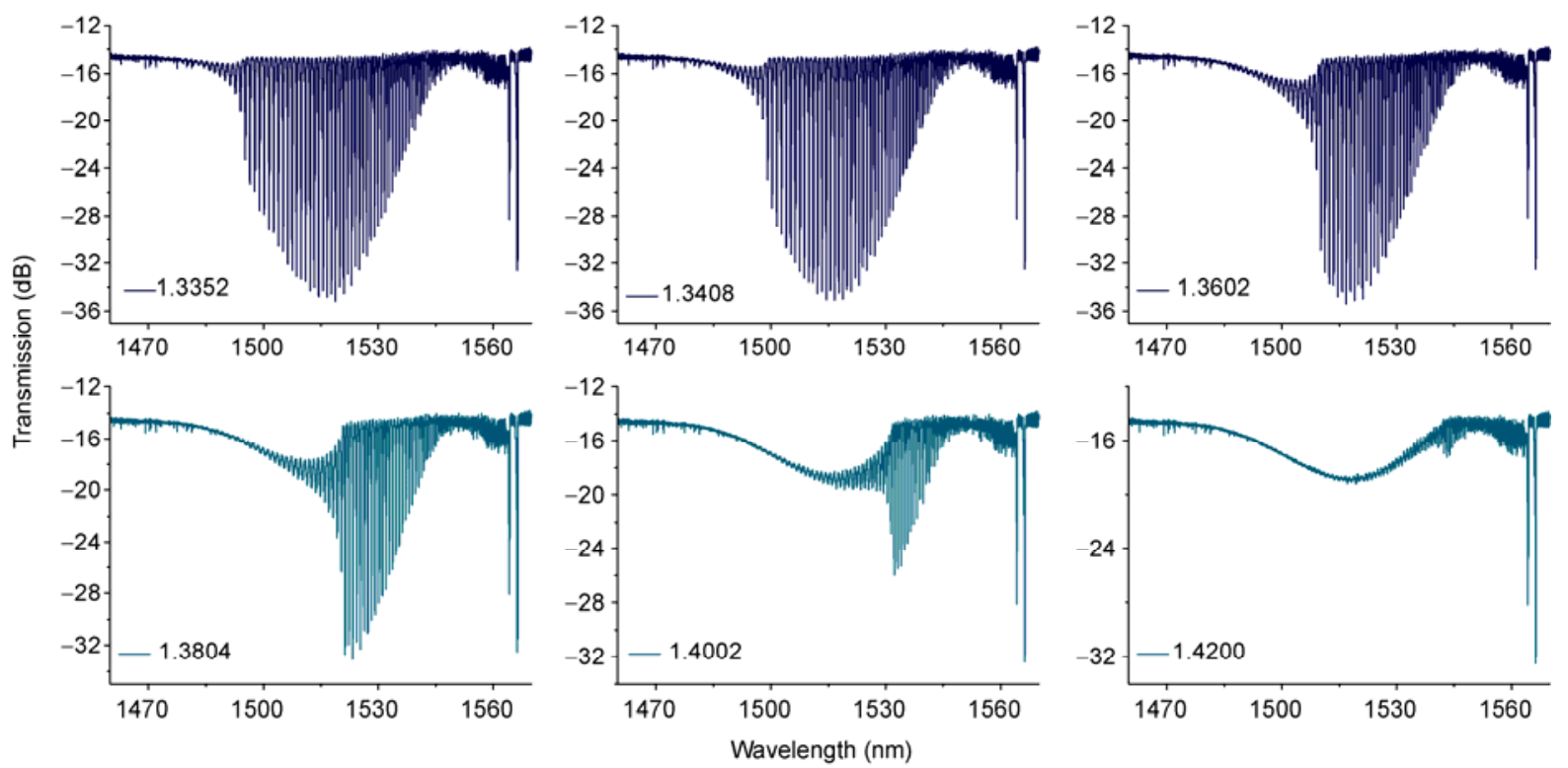

Figure 3 Transmission spectra of $10^{\circ}$ TFBG versus sucrose solutions.

RI are measured. It presents the TFBG transmission spectra for several values of $n_{s}$ (RI measured by an Abbe refractometer) ranging from 1.3352 to 1.4200 in sucrose solutions. Just as we expect that the high order cladding modes shift notably but the lower order modes and the core mode are not affected. It is clear that the cut-off cladding mode resonance depending on the penetration of the evanescent field of the cladding modes into the outside shifts toward long-wavelength for the increase of SRI. This cut-off cladding mode can be used to measure media RI without ambiguity from neighboring resonances. As shown in Figure 4, we obtain the relative resonance position by detecting the $\lambda_{\text {cut-off }}$ as a function of $n_{s}$. A linear response with RI sensitivity up to $557.79 \mathrm{~nm} / \mathrm{RIU}$ has been experimentally achieved, which identifies its feasibility as a high sensitive fiber-optic refractometer for large range RI measurement.

\subsection{Intracellular density of cells measurement}

Figure 5 shows the transmission spectra of $10^{\circ}$ TFBG under different cell sample suspensions (i.e. leukemia cell S50 and $\mathrm{S} 60$, both of them with the same concentrations of $2 \times$ $10^{5} / \mathrm{mL}$ ), the buffer solution (saline RI $\sim 1.33$ ) and ethanol (RI 1.36) used for comparison. It highlights the fact that, when the surrounding RI increases (from saline $\sim 1.33$ to

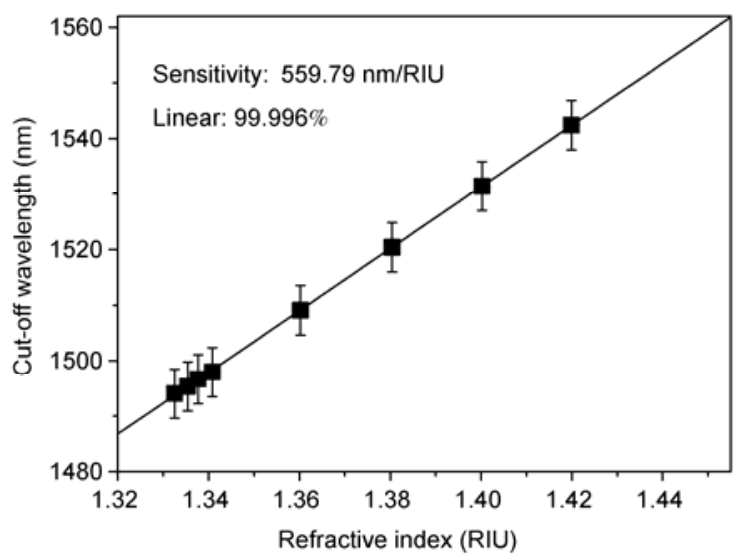

Figure 4 Linear response between the wavelength of TFBG cut-off resonance and SRI changes.

ethanol $\sim 1.36$ ), the cladding mode resonances gradually disappear for the modes becoming leaky, and the cut-off wavelength (as marked by arrows) shifts towards longer wavelengths in easily distinguishable fashion. However, for the small RI changes associated with the biochemical solutions S50 and S60, it is hard to discriminate them directly in transmission. On the other hand, if we calculate the spectral intensity difference of these cases versus saline as shown in 


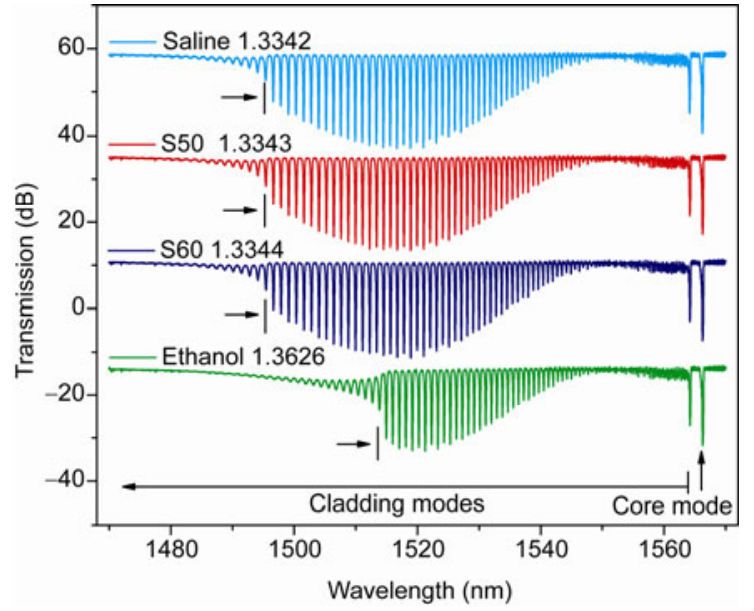

Figure 5 Transmission spectra of $10^{\circ}$ TFBG versus different sample suspensions.

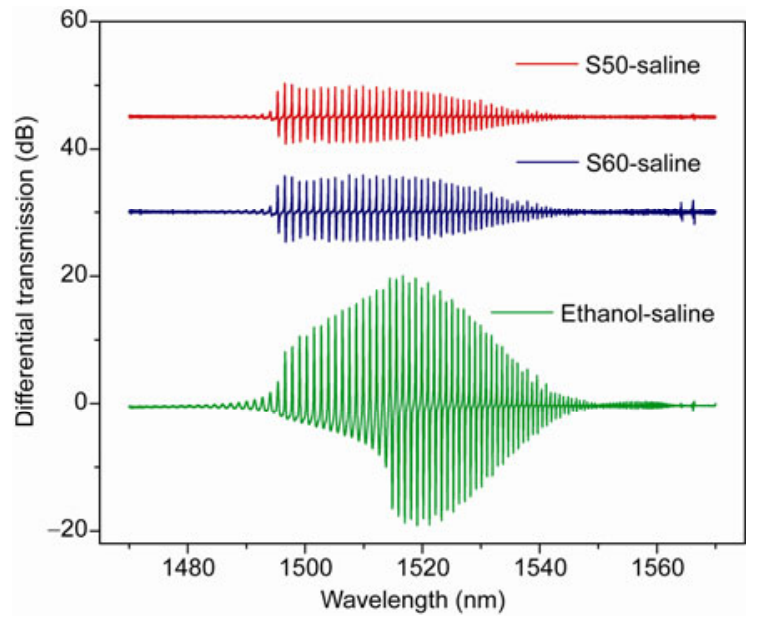

Figure 6 Differential transmission of cell samples versus saline.

Figure 6, the slight RI perturbations induce clearly measurable amplitude changes. This is because changes in the RI of the sample solutions shift the cladding mode resonances by small amounts but they have such large slopes that the differential spectra then show significant amplitude changes. Figures 6 and 7 experimentally show that $\mathrm{S} 60$ has slightly higher RI than S50, which agrees with fact that S60 contains "young" leukemia cells with a higher intracellular density. An additional feature of the TFBG is temperature self-calibration where spectral shifts due to temperature changes can be eliminated by referencing all wavelengths to the core mode [1], which is unaffected by the surrounding RI and has the same temperature dependence with the other modes, as shown in Figure 7, thereby ensuring that the differential spectra are due solely to RI changes.

\section{Conclusions}

The feasibility of $10^{\circ}$ TFBG based fiber sensing probe for

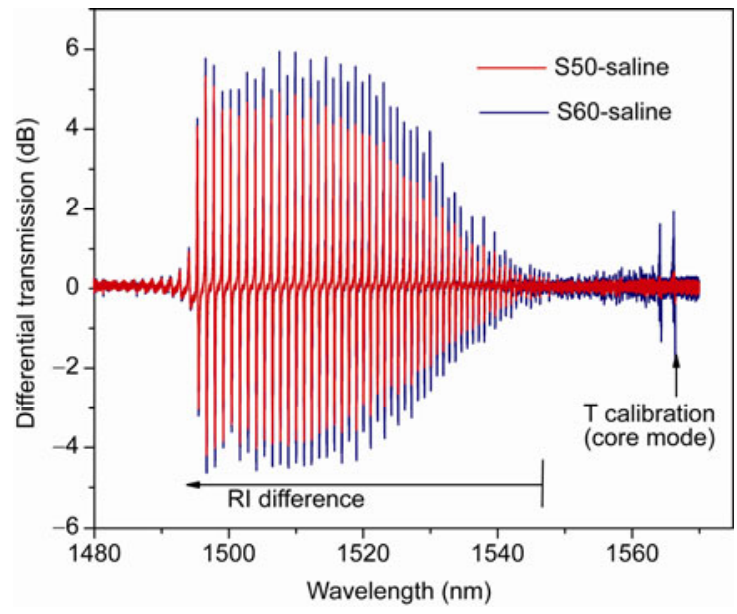

Figure 7 Temperature-self-calibrated cell-sample discrimination via RI insensitive core mode reference.

high-sensitive cell sample suspension measurement has been demonstrated. Human acute leukemia cells with different intracellular densities were clearly discriminated by identifying their slight refraction index (RI) perturbations in the range from 1.3342 to 1.3344 , combining with a temperature self-calibration property. The extremely high sensitivity for water-like solutions together with the temperature-self-calibration ability makes it a good candidate for biological samples discrimination in a stable and label-free way.

This work was supported by the National Natural Science Foundation of China (61205080, 61235005 and 61036012), the National Innovation Fund for Technology (2C26214405312), Guangdong Natural Science Foundation of China (S201101000163 and S2012010008385), the Specialized Research Fund for the Doctoral Program of Higher Education of China (20114401120006), the Pearl River Scholar for Distinguished Young Scientist (2011J2200014). J. Albert acknowledges the support of the Natural Sciences and Engineering Research Council of Canada (NSERC) and the Canada Research Chair Program.

1 Albert J, Shao L Y, Caucheteur C. Tilted fiber bragg grating sensors. Laser Photonic Rev, 2013, 7: 83-108

2 Lu Y C, Huang W P, Jian S S. Full vector complex coupled mode theory for tilted fiber gratings. Opt Express, 2010, 18: 713-725

3 Erdogan T, Sipe J E. Tilted fiber phase gratings. J Opt Soc Am A, 1996, 13: 296-313

4 Lee K S, Erdogan T. Transmissive tilted gratings for LP01-to-LP11 mode coupling. IEEE Photon Technol Lett, 1999, 11: 1286-1288

5 Laffont G, Ferdinand P. Tilted short-period fiber-Bragg-grating induced coupling to cladding modes for accurate refractometry. Mea Sci Technol, 2001, 12: 765-770

6 Chen C, Albert J. Strain-optic coefficients of individual cladding modes of single mode fibre: Theory and experiment. Electron Lett, 2006, 42: 1027-1028

7 Caucheteur C, Bette S, Chen C, et al. Tilted fiber Bragg grating refractometer using polarization-dependent loss measurement. IEEE Photon Technol Lett, 2008, 20: 2153-2155

8 Guo T, Chen C K, Laronche A, et al. Power-referenced and temperature-calibrated optical fiber refractometer. IEEE Photon Technol Lett, 2008, 20: 635-637

9 Guo T, Tam H Y, Krug P A, et al. Reflective tilted fiber Bragg grating refractometer based on strong cladding to core recoupling. Opt Express, 2009, 17: 5736-5742 
10 Li T, Dong X, Chan C C, et al. Power-referenced optical fiber refractometer based on a hybrid fiber grating. IEEE Photon Technol Lett, 2011, 23: 1706-1708

11 Lu Y C, Rui G, Wang C C, et al. Polarization effects in tilted fiber Bragg grating refractometers. J Lightwave Technol, 2010, 28: 16771684

12 Chan C F, Chen C, Jafari A, et al. Optical fiber refractometer using narrowband cladding-mode resonance shifts. Appl Opt, 2007, 46: 1142-1149

13 Zhang L, Yang S, He Y J, et al. Fluorouracil selectively enriches stem-like leukemic cells in a leukemic cell line. Int J Biol Sci, 2010, 5: 419-427

14 Stong R C, Korsmeyer S J, Parkin J L, et al. Human acute leukemia cell line with the $t(4 ; 11)$ chromosomal rearrangement exhibits $B$ lineage and monocytic characteristics. Blood, 1985, 65: 21-31

15 Srivastava B I, Wright J J, Bakhshi A. Immunoglobulin chain gene rearrangements in a $t(4 ; 11)$ acute leukaemia with monocytoid blasts. Br J Haematol, 1986, 63: 321-329

16 Rowley J D, Diaz M O, Espinosa R, et al. Mapping chromosome band 11q23 in human acute leukemia with biotinylated probes: Identification of $11 \mathrm{q} 23$ translocation breakpoints with a yeast artificial chromosome. Proc Natl Acad Sci USA, 1990, 87: 9358-9362

17 Kobayashi H, Takemura Y, Miyachi H. Molecular characterization of human acute leukemia cell line resistant to ZD9331, a nonpolyglutamatable thymidylate synthase inhibitor. Cancer Chemother Pharmacol, 1998, 42: 105-110

18 Liu B, Zhao L, Ma H Z. Knockdown of MRP4 by lentivirus-mediated siRNA improves sensitivity to adriamycin in adriamycin-resistant acute myeloid leukemia cells. Chin Sci Bull, 2012, 57: 90-97

19 Tajima M, Tamura H, Maejima K. Method of separating mouse platelets by discontinuous sucrose-gradient centrifugation. Jikken Dobutsu, 1987, 36: 79-81

20 Tajima M, Tamura H, Maejima K. Method of separating mouse platelets by discontinuous sucrose-gradient centrifugation. Jikken Dobutsu, 1987, 36: 79-81

21 Liu Y, Wei J G, Wu B B, et al. Density alteration in non-physiological cells. Nature Precedings, http://hdl.handle.net/10101/npre.2011.6541.1

22 Liu Y, Tortora G, Ryan M, et al. Potato dextrose agar antifungal susceptibility testing for yeasts and molds: Evaluation of phosphate effect on antifungal activity of CMT-3. Antimicrob Agent Chemother, 2002, 5: 1455-1461

Open Access This article is distributed under the terms of the Creative Commons Attribution License which permits any use, distribution, and reproduction in any medium, provided the original author(s) and source are credited. 